\title{
Cumulative prospect theory and the St. Petersburg paradox
}

\section{Journal Article}

Author(s):

Oliver, Marc; Wang, Mei

Publication date:

2006-08

Permanent link:

https://doi.org/10.3929/ethz-b-000032060

Rights / license:

In Copyright - Non-Commercial Use Permitted

Originally published in:

Economic Theory 28(3), https://doi.org/10.1007/s00199-005-0641-6 


\title{
Cumulative prospect theory and the St. Petersburg paradox $\star$
}

\author{
Marc Oliver Rieger ${ }^{1}$ and Mei Wang ${ }^{2}$ \\ 1 University of Zürich, Mathematical Institute, Winterthurerstr. 190, 8057 Zürich, SWITZERLAND \\ (e-mail: rieger@math.unizh.ch) \\ 2 ETH Zürich, Institute of Economics Research, Weinbergstr. 35, 8092 Zürich, SWITZERLAND \\ (e-mail: meiwang@wif.gess.ethz.ch)
}

Received: October 14, 2004; revised version: May 6, 2005

Summary. We find that in cumulative prospect theory (CPT) with a concave value function in gains, a lottery with finite expected value may have infinite subjective value. This problem does not occur in expected utility theory. The paradox occurs in particular in the setting and the parameter regime studied by Tversky and Kahneman [15] and in subsequent works. We characterize situations in CPT where the problem can be resolved. In particular, we define a class of admissible probability distributions and admissible parameter regimes for the weighting- and value functions for which finiteness of the subjective value can be proved. Alternatively, we suggest a new weighting function for CPT which guarantees finite subjective value for all lotteries with finite expected value, independent of the choice of the value function. Some of these results have already been found independently by Blavatskyy [4] in the context of discrete lotteries.

Keywords and Phrases: Cumulative prospect theory, Probability weighting function, St. Petersburg paradox.

JEL Classification Numbers: C91, D81.

* We thank Pavlo Blavatskyy and Thorsten Hens for their helpful remarks regarding our paper. Moreover, we thank the referee for his constructive suggestions. This research was supported by the University Research Priority Program "Finance and Financial Markets" a research instrument of the University of Zürich.

Correspondence to: M. Wang 


\section{Introduction}

\subsection{Review of Cumulative Prospect Theory (CPT)}

Expected utility theory has been the foundation for most of our modern economic theories on uncertainty and risk. However, it has been challenged by more and more empirical results pointing at violations of it. For example, it has been found that people tend to think of the outcome as a relative change rather than the final status, they have different risk attitudes towards gains and losses, and they tend to overweight unlikely events but underweight highly possible events.

All these observations call for developments of alternative theories that are psychologically more appealing and descriptively more valid. The Cumulative Prospect Theory (CPT), introduced by Tversky and Kahneman [15], stands out as one of the most well-accepted descriptive alternatives to expected utility theory. It has three important features:

1. Instead of evaluating the final wealth, the payoffs are framed as gains or losses as compared to some reference point.

2. The loss looms larger than the gain, hence the value function in losses is steeper than the value function in gains.

3. A weighting function, in which the small probabilities are underweighted and the moderate to large probabilities are overweighted, is introduced to transform the cumulative probability distribution.

The first two features are reflected in the two-part S-shaped value functionconcave in gains and convex in losses. The prototypical example has been given in [15] for $\alpha, \beta \in(0,1)$ and $\lambda>1$ :

$$
u(x):=\left\{\begin{array}{cl}
x^{\alpha}, & x \geq 0 \\
-\lambda(-x)^{\beta}, & x<0 .
\end{array}\right.
$$

We mention that the distinction between $\alpha$ and $\beta$ is not essential to our results.

The third feature is captured by weighting the (cumulative) probability distribution by an S-shaped function, the so-called weighting function $w$. The original example of [15] is given by

$$
w(F):=\frac{F^{\gamma}}{\left(F^{\gamma}+(1-F)^{\gamma}\right)^{1 / \gamma}} .
$$

It is possible to assign different weighting functions for gains and losses (denoted by $w_{+}$and $w_{-}$), and we will use $\delta$ to denote the parameter in losses. Our results extend to a large class of possible weighting functions, in particular including the aforementioned and the alternative weighting function

$$
w(F):=\exp \left(-(-\ln (F))^{\gamma}\right)
$$

for $\gamma \in(0,1)$, which has been suggested by Prelec [13]. 
We define the subjective utility by

$$
U(p):=\int_{-\infty}^{0} u(x) \frac{d}{d x}\left(w_{-}(F(x))\right) d x+\int_{0}^{+\infty} u(x) \frac{d}{d x}\left(w_{+}(F(x))\right) d x,
$$

where $F(x):=\int_{-\infty}^{x} d p$. This is a generalization of the original formulation in [15]. The generalization allows for arbitrary (continuous) outcomes, and not only for discrete values. Our formulation includes in particular the discrete case of [15]. This can be seen easily by setting $p(x):=\sum_{i} p_{i} \delta_{x_{i}}$, where $\delta_{x}$ is a Dirac mass at $x$, the probabilities $p_{i}>0$ satisfy $\sum_{i} p_{i}=1$, and the (discrete) outcomes are given by the real numbers $x_{i}$. The formula for $U$ in the discrete setting then becomes

$$
U(p):=\sum_{i=-m}^{0} v\left(x_{i}\right) \pi_{i}^{-}+\sum_{i=0}^{n} v\left(x_{i}\right) \pi_{i}^{+},
$$

where $\pi_{n}^{+}=w_{+}\left(p_{n}\right), \pi_{i}^{+}=w_{+}\left(p_{i}+\cdots+p_{n}\right)-w_{+}\left(p_{i+1}+\cdots+p_{n}\right)$ for $0 \leq i \leq n-1$ and accordingly for the $\pi_{i}$ with $m \leq i<0$. (Here the possible outcomes are denoted by $x_{i}$ and $x_{-m}<\cdots<x_{-1}<x_{0}=0<x_{1}<\cdots<x_{n}$. For details see [15].)

The classical formulation of the St. Petersburg problem (as a discrete lottery) would correspond to this special case. Nevertheless, we prefer to take a little extra effort to use the more general continuous setting. Readers who are not familiar with the continuous formulation may just replace all integrals by sums to arrive at the more usual discrete case.

\subsection{A remark on the monotonicity of the weighting function}

The weighting function $w$ is usually assumed to be a strictly increasing function. This follows from the basic fact that people weigh higher probabilities stronger than lower probabilities. However, to our knowledge it hasn't been pointed out so far that the oldest and most widely used form of the weighting function

$$
w(F):=\frac{F^{\gamma}}{\left(F^{\gamma}+(1-F)^{\gamma}\right)^{1 / \gamma}},
$$

as suggest by [15], does not satisfy this condition for all $\gamma \in(0,1)$. In fact, numerical computations show that the function $w$ is partially decreasing for $\gamma \leq 0.278$, compare Figure 1. The problem disappears for larger values of $\gamma$. It is surprising that it hasn't been found earlier (to our knowledge). This can only be explained with the analytical difficulties which the complicated structure of $w$ poses.

Other weighting functions, in particular the ones defined by [13] and [11] are strictly increasing for all values of $\gamma \in(0,1)$. This observation seems to suggest that for experimental studies alternative forms should be preferred over the original form of (2). The problem, however, is not too severe, since previous studies mostly measured values of $\gamma \geq 0.3$, and in this parameter regime, the weighting function $w$ seems indeed to be strictly increasing as numerical computations suggest. 


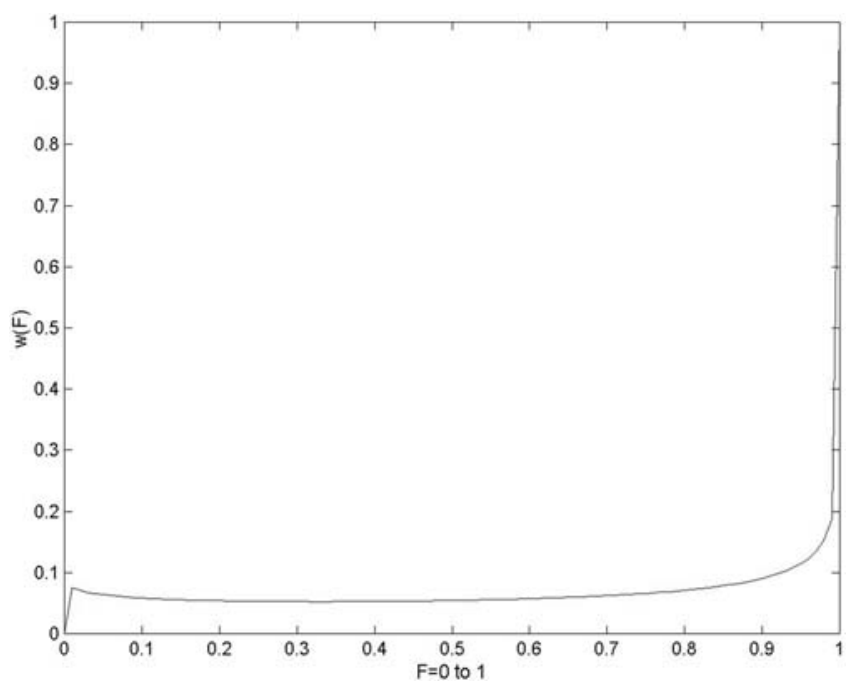

Figure 1. The classical weighting function defined in (2) and introduced by [15] is not monotone for small values of $\gamma$. (Here: $\gamma=0.2$ )

In fact, one can prove that $w$ is monotone for $\gamma \geq 0.5$ : consider the derivative of $\ln (w(F))$ with respect to $F$. An easy computation shows that

$$
\frac{d}{d F}\left(\ln (w(F))=\frac{(\gamma-1) F^{\gamma}+(\gamma+F) \gamma(1-F)^{\gamma-1}}{F\left(F^{\gamma}+(1-F)^{\gamma}\right)},\right.
$$

and hence $w$ is monotone if and only if $H_{\gamma}(F):=(\gamma-1) F^{\gamma}+(\gamma+F) \gamma(1-F)^{\gamma-1}$ is positive for all $F \in[0,1]$. Since the first summand can be estimated from below by $\gamma-1$ and the second by $\gamma$, this is the case for all $\gamma \geq 0.5$.

On the other hand, evaluating $H_{\gamma}(F)$, e.g., for $\gamma=0.25$ and $F=0.1$ gives a negative value for $H_{\gamma}(F)$ and hence one can prove that $w$ is non-monotone for $\gamma \leq 0.25$.

In our paper we will nevertheless occasionally use the weighting function $w$ as given by (2), since it is the most frequently studied version, and the problems we are concerned with are independent of the non-monotonicity for small values of the parameter $\gamma$. Our main motivation, however, is to provide general results covering all classes of weighting functions, hence the function $w$ will only be a specific example for us.

\subsection{The classical St. Petersburg paradox}

The St. Petersburg paradox is usually explained with the following example: the player Paul is reluctant to pay enormous amounts of money for a gamble that Peter offers him - he will get $2^{i}$ ducats when the coin lands "heads" on the ground for the first time at the $i$ th throw - which has an infinitely large expected value (EV). This example already dates back to Bernoulli [3]. The solution of this problem is 
usually to replace the formula of EV with the one of expected utility, in which a strictly concave utility function makes the subjective utility of the large outcome no longer high enough to compensate the very low probability associated with it.

It is, however, important to keep in mind that for gambles with infinite $\mathrm{EV}$, the strict concavity of the utility function alone cannot guarantee the expected utility to be finite. For example, if Peter offers Paul $2^{2 i}$ ducats when the coin lands "heads" at the $i$-th throw, then with a strictly concave utility function like $u(x):=x^{0.8}$, the expected utility is still infinitely large. (Even in the original example, the strictly concave utility function $u(x):=x-e^{-x}$ still leads to an infinite expected utility.) Such insight was first made by Menger [12] with his illustration of the "SuperPetersburg Paradox". He concluded that unless the utility function is bounded, it is impossible to discriminate all possible probability distributions. One could argue, however, it is not necessary to discriminate all possible probability distributions, because no individuals or organizations can offer a lottery with unlimited EV. Actually, Arrow [2] pointed out that if we only consider distributions with finite $\mathrm{EV}$, we can still guarantee finite expected utility even though the utility function is unbounded. More precisely he found the following result:

Proposition 1 Let $p$ be a probability measure with finite $E V E(p)<\infty$ and let $u: \mathbb{R} \rightarrow \mathbb{R}$ be a strictly increasing, concave utility function, then the utility $U(p):=$ $\int u d p$ is finite.

(We remark again that this statement is a generalization of the case of discrete outcomes where the integral is replaced by a sum. Hence the usual formulation of the St. Petersburg problem in terms of a discrete lottery is included.)

In other words, the St. Petersburg paradox can be resolved by allowing only for "realistic" lotteries, i.e., under the assumption of a finite EV, a (not necessarily strictly) concave utility function is sufficient to guarantee that the expected utility is finite.

Even though this fundamental statement is mathematically easy in the framework of expected utility theory, it turns out to be false in the context of CPT. In fact, we will show in the Section 2 that in cumulative prospect theory a gamble with a finite $\mathrm{EV}$ can have an infinite subjective value - independent of the concavity of the value function $u$.

The intuitive explanation for this phenomenon can be seen as follows: In expected utility theory, a concavity of the utility function is sufficient to bound the utility as long as the EV of the lottery is finite, because a person's certainty equivalent is less than the EV if he/she is risk averse. When the probability weighting is nonlinear, however, the risk attitude depends on the interaction of the value and weighting function, as well as the probability distribution $[9,16]$. In case of the probability weighting function as formulated in CPT [15], the infinite slope of the weighting function at zero and unity counteracts with the concave value function when a lottery has a too long "tail" similar to the St. Petersburg gamble, the proportional weights given to the outcomes at the tails are unboundedly high. As a result, an individual's subjective value for a lottery with a finite EV can be unbounded even if his/her value function is concave. This is not a very reasonable situation for individual decision making. To avoid this problem, we need to make sure that 
the interaction of weighting function and value function behaves like the concave utility function in expected utility theory at the tails of probability distribution.

Generalizing this idea, we find special cases in CPT under which the problem can be resolved. In particular we will define a class of admissible probability distributions and admissible parameter regimes (Sect. 2.3) and we will suggest an alternative weighting function which allows for an extension of Theorem 1 to CPT (Sect. 2.4). Some of the results presented in Section 2 have already been found independently by Blavatskyy in the context of discrete lotteries [4].

In the final Section 3 we summarize our results and discuss the possible modifications of CPT that resolve the paradox.

\section{The St. Petersburg paradox in CPT}

\subsection{General result}

The central result of this section is the following Theorem 1. It provides precise conditions under which the St. Petersburg paradox in CPT occurs. We will later derive several results from this theorem which are more accessible and which cover situations of particular interest, compare Theorems 3-5.

Theorem 1 (General result) Let $U$ be a CPT subjective utility given by

$$
U(p):=\int_{-\infty}^{0} u(x) \frac{d}{d x}\left(w_{-}(F(x))\right) d x+\int_{0}^{+\infty} u(x) \frac{d}{d x}\left(w_{+}(F(x))\right) d x,
$$

where the value function $u$ is continuous, monotone, convex for $x<0$ and concave for $x>0$. Assume that there exist constants $\alpha, \beta \geq 0$ such that

$$
\lim _{x \rightarrow+\infty} \frac{u(x)}{x^{\alpha}}=u_{1} \in(0,+\infty), \quad \lim _{x \rightarrow-\infty} \frac{|u(x)|}{|x|^{\beta}}=u_{2} \in(0,+\infty),
$$

and that the weighting functions $w_{ \pm}$are continuous, strictly increasing functions from $[0,1]$ to $[0,1]$ such that $w_{ \pm}(0)=0$ and $w_{ \pm}(1)=1$. Moreover assume that $w_{ \pm}$are continuously differentiable on $(0,1)$ and that there are constants $\delta, \gamma>0$ such that

$$
\lim _{y \rightarrow 0} \frac{w_{-}^{\prime}(y)}{y^{\delta-1}}=w_{1} \in(0,+\infty), \quad \lim _{y \rightarrow 1} \frac{1-w_{+}^{\prime}(y)}{(1-y)^{\gamma-1}}=w_{2} \in(0,+\infty) .
$$

Let $p$ be a probability distribution with $E(p)<\infty$. Then $U(p)$ is finite if $\alpha<\gamma$ and $\beta<\delta$. This condition is sharp as can be seen from Theorem 2 below.

In particular, the subjective utility may be infinite for distributions with finite $\mathrm{EV}$ in the setting and the parameter range of the original work by Tversky and Kahneman [15] where $\alpha \approx 0.88$ and $\gamma \approx 0.65$ and hence the (sharp) condition $\alpha<\gamma$ is violated, compare Section 2.2.

We will present a proof of the general result Theorem 1 together with some extensions in Section 2.3. Before that, we give an explicit example for a probability distribution of outcomes which has a finite EV, but an infinite subjective utility in the setting of Tversky and Kahneman [15]. A similar example has already been pointed out by Blavtskyy [4]. 
Theorem 2 (Finite EV, but infinite subjective utility) Let $\gamma, \alpha \in(0,1), q>2$. Let the probability density $p$ of possible outcomes be given by

$$
p(x):= \begin{cases}0, & x \leq 1 \\ C x^{-q}, & x>1\end{cases}
$$

where $C:=\int_{0}^{\infty} x^{-q} d x$.

Let the weighting function $w_{+}:[0,1] \rightarrow[0,1]$ be given by (2) and the value function on $u: \mathbb{R}_{+} \rightarrow \mathbb{R}$ be given by (1), i.e. value- and weighting function are chosen as in the original work by Tversky and Kahneman [15]. Let $F(x):=\int_{-\infty}^{x} p(x) d x$ be the total probability for an outcome less than $x$. Then we have $E(p)<+\infty$ and $u$ strictly concave, but for $\alpha>\gamma$ and $q$ sufficiently close to 2 the subjective utility is infinite, i.e.

$$
U(p):=\int u(x) \frac{d}{d x}(w(F(x))) d x=+\infty .
$$

This result shows that it is not possible to resolve the St. Petersburg paradox in the framework of CPT in the same way as in the utility theory: Even if we assume strict concavity of the value function (corresponding to a risk-averse behavior) and a finite EV for the probability distribution of outcomes (thus excluding unrealistic situations with infinite average outcome), the subjective utility can still be infinite! This paradox has already been observed independently by Blavatskyy in the context of discrete lotteries. We refer the reader to his article [4] for details.

It should be mentioned that the problem does not arise from the convex-concave structure of the value function in CPT, since in our example we have only positive outcomes (i.e., we work only in the concave part). It does also not arise from a specific choice of the weighting function, since we have chosen the standard form already introduced by [15], and could as well use an alternative form as suggested, e.g., in [13]. This can also be seen from the general conditions under which the problem occurs as stated in Theorem 1 above.

Let us now have a look at the proof of Theorem 2 which illustrates best the role that the weighting function plays in the paradox.

Proof of Theorem 2. First we prove that $E(p)<+\infty$ using that $q>2$ :

$$
E(p)=\int_{1}^{\infty} x p(x) d x=\int_{1}^{\infty} x^{-q+1} d x=\frac{1}{2-q}<+\infty .
$$

The concavity of $u$ on $\mathbb{R}_{+}$is clear from the definition, so we only need to show that the utility $U(p)$ is infinite. We compute $F(x)=\int_{1}^{x} p(x) d x=\frac{1}{q+1}\left(1-x^{1-q}\right)$. Denote $C:=\frac{1}{q+1} \in\left(0, \frac{1}{3}\right)$. Now we can calculate $U(p)$ :

$$
\begin{aligned}
U(p) & =\int u(x) \frac{d}{d x}(w(F(x))) d x \\
& =\int_{1}^{\infty} x^{\alpha} \frac{d}{d x}\left(\frac{F(x)^{\gamma}}{\left(F(x)^{\gamma}+(1-F(x))^{\gamma}\right)^{1 / \gamma}}\right) d x
\end{aligned}
$$




$$
\begin{aligned}
= & \int_{1}^{\infty} x^{\alpha} \frac{d}{d x}\left(\frac{C^{\gamma}\left(1-x^{1-q}\right)^{\gamma}}{\left(C^{\gamma}\left(1-x^{1-q}\right)^{\gamma}+(1-C)^{\gamma} x^{(1-q) \gamma}\right)^{1 / \gamma}}\right) d x \\
= & \int_{1}^{\infty} C^{\gamma}\left[\gamma\left(1-x^{1-q}\right)^{\gamma-1} x^{\alpha-q}(q-1)\right. \\
& \left(C^{\gamma}\left(1-x^{1-q}\right)^{\gamma}+(1-C)^{\gamma} x^{\gamma-q \gamma}\right)^{-1 / \gamma} \\
& +\left(1-x^{1-q}\right)^{\gamma}\left(C^{\gamma}\left(1-x^{1-q}\right)^{\gamma}+(1-C)^{\gamma} x^{\gamma-q \gamma}\right)^{-\frac{1}{\gamma}-1} \\
& \left.\left(C^{\gamma}\left(1-x^{1-q}\right)^{\gamma-1} x^{\alpha-q}-(1-C)^{\gamma}(1-q) x^{\gamma+\alpha-q \gamma-1}\right)\right] d x .
\end{aligned}
$$

Now we prove the following estimates for positive numbers $c_{1}, c_{2}$ :

$$
\begin{aligned}
\left(1-x^{1-q}\right)^{\gamma-1} & \geq 1 \\
\left(c_{1}\left(1-x^{1-q}\right)^{\gamma}+c_{2} x^{\gamma-q \gamma}\right)^{-1 / \gamma} & \geq\left(c_{1}+c_{2}\right)^{-1 / \gamma} . \\
\left(c_{1}\left(1-x^{1-q}\right)^{\gamma}+c_{2} x^{\gamma-q \gamma}\right)^{-1 / \gamma-1} & \geq\left(c_{1}+c_{2}\right)^{-1 / \gamma-1} .
\end{aligned}
$$

Inequality (6) simply follows from $1-x^{1-q} \leq 1$ and $\gamma-1 \in(-1,0)$, whereas (7) and (8) follow from $\left(1-x^{1-q}\right)^{\gamma} \leq 1$ and $x^{\gamma-q \gamma} \leq 1$. (Here we use that $x \geq 1$.)

We apply these inequalities to (5) with $c_{1}:=C^{\gamma}$ and $c_{2}:=(1-C)^{\gamma}$ to derive

$$
\begin{aligned}
U(p) \geq & \int_{1}^{\infty} C^{\gamma} \gamma(q-1)\left(C^{\gamma}+(1-C)^{\gamma}\right)^{-1 / \gamma} x^{\alpha-q} \\
& +C^{2 \gamma}\left(1-x^{1-q}\right)^{\gamma}\left(C^{\gamma}+(1-C)^{\gamma}\right)^{-1 / \gamma-1} x^{\alpha-q} \\
& +C^{\gamma}(1-C)^{\gamma}(q-1) x^{\alpha+\gamma-q \gamma-1} d x .
\end{aligned}
$$

We use that for $x \geq 2$ we have $\left(1-x^{1-q}\right)^{\gamma} \geq\left(1-2^{1-q}\right)^{\gamma}$ and since $q>2$ we even have $\left(1-x^{1-q}\right)^{\gamma} \geq\left(1-2^{-1}\right)^{\gamma}=2^{-\gamma}$. Furthermore we estimate the integral in (9) by the integral from 2 to $+\infty$, using that the integrant is positive:

$$
\begin{aligned}
U(p) \geq & \int_{2}^{\infty} C^{\gamma} \gamma(q-1)\left(C^{\gamma}+(1-C)^{\gamma}\right)^{-1 / \gamma} x^{\alpha-q} \\
& +C^{2 \gamma} 2^{-\gamma}\left(C^{\gamma}+(1-C)^{\gamma}\right)^{-1 / \gamma-1} x^{\alpha-q} \\
& +C^{\gamma}(1-C)^{\gamma}(q-1) x^{\alpha+\gamma-q \gamma-1} d x .
\end{aligned}
$$

Writing $K=C^{\gamma}+(1-C)^{\gamma}$ and collecting terms with the same expression in $x$ we arrive at

$$
\begin{aligned}
U(p) \geq & \int_{2}^{\infty} C^{\gamma}\left(\gamma(q-1) K^{-1 / \gamma}+C^{\gamma} 2^{-\gamma} K^{-1 / \gamma-1}\right) x^{\alpha-q} \\
& +(q-1) C^{\gamma}(1-C)^{\gamma} x^{\alpha+\gamma-q \gamma-1} d x .
\end{aligned}
$$

We recall that a function $x^{s}$ is integrable on $(2,+\infty)$ if and only if $s<-1$, hence the first term in this integral is integrable if and only if $\alpha-q<-1$ which is always the case by the assumptions $\alpha<1$ and $q>2$. However, the second term is only integrable if $\alpha+\gamma-q \gamma<0$. Since we can choose $q$ arbitrarily close to 2 , this is only the case if $\alpha<\gamma$. In other words, if we choose $q$ close to 2 and $\alpha>\gamma$, e.g. $q:=3, \alpha:=3 / 4, \gamma:=1 / 4$, then (10) becomes $+\infty$ and we have proved that $U(p)=+\infty$. 


\subsection{Results on finite utility from CPT}

We have seen in the previous section that in standard CPT the St. Petersburg paradox cannot be resolved. However, there are specific situations in which the problem does not occur and which can be derived from the general results of Theorem 1 . In this section we will discuss such situations.

We first consider conditions on the probability distribution of the outcomes. The St. Petersburg paradox can obviously not occur if we restrict ourselves to a finite set of possible outcomes, but even a bounded set of possible outcomes suffices to prevent infinite utility:

Theorem 3 (Finiteness for special distributions I) Let $U$ be a CPT subjective utility functional and $p$ be a probability distribution with bounded support, i.e. $\operatorname{supp} p:=\overline{\{x \in \mathbb{R} ; p(x)>0\}} \subset[a, b]$, where $a>-\infty$ and $b<+\infty$. Then $U(p)$ is finite.

Proof. This follows from the general result, Theorem 1, but it can also be seen directly. We assume for simplicity that $w:=w_{-}=w_{+}$, use the monotonicity of $u$ and that $w(F) \in[0,1]$ :

$$
\begin{aligned}
U(p) & =\int_{a}^{b} u(x) \frac{d}{d x} w(F(x)) d x \leq u(b) \int_{a}^{b} \frac{d}{d x} w(F(x)) d x \\
& =u(b)(w(F(b))-w(F(a))) \leq u(b)<\infty .
\end{aligned}
$$

Many interesting probability distributions (e.g. normal Gauss distributions) do not have a bounded support. Therefore the following extension is useful:

Theorem 4 (Finiteness for special distributions II) Let $U$ be a CPT subjective utility functional and $p$ be a probability distribution with exponential decay at $+\infty$, i.e. there exist $a, b, c>0$ such that $p(x) \leq a e^{-b x}$ for all $x \geq c$. Moreover, assume that (4) holds. Then $U(p)<+\infty$. (The corresponding condition for $-\infty$ would ensure that $U(p)>-\infty$.)

Proof. This result is an immediate consequence of Theorem 6.

If one wants to allow for arbitrary probability distributions, a general finiteness result can be given for bounded value functions:

Theorem 5 (Bounded value functions) Let $U$ be a CPT subjective utility functional with bounded value function $|u(x)| \leq C$ and let $p$ be a probability distribution. Then $U(p)$ is finite.

Proof. Again, this is a corollary of Theorem 1, but a direct proof, following the ideas of the proof of Theorem 3 is also easy.

Under the restriction to finite EVs, one can also obtain finiteness if the constant of the value function is smaller than the parameter of the weighting function, i.e. $\max (\alpha, \beta)<\gamma$, as we will show in Section 2.3. (In a certain sense, the value 
Table 1. Experimental values of $\alpha, \beta$ and $\gamma, \delta$ from various studies, compare (3) and (4) for the precise definition of $\alpha, \beta, \gamma, \delta$

\begin{tabular}{lccc}
\hline Study & $\begin{array}{c}\text { Estimate } \\
\text { for } \alpha, \beta\end{array}$ & $\begin{array}{c}\text { Estimate } \\
\text { for } \gamma, \delta\end{array}$ & $\begin{array}{c}\alpha<\gamma \\
\beta<\delta\end{array}$ \\
\hline $\begin{array}{l}\text { Tversky and Kahneman [15] } \\
\text { gains: } \\
\text { losses: }\end{array}$ & 0.88 & 0.61 & no \\
\hline Camerer and Ho [6] & 0.88 & 0.69 & no \\
\hline $\begin{array}{l}\text { Tversky and Fox [14] } \\
\text { Wu and Gonzalez [18] }\end{array}$ & 0.37 & 0.56 & yes \\
\begin{tabular}{l} 
gains: \\
\hline $\begin{array}{l}\text { Abdellaoui [1] } \\
\text { gains: }\end{array}$
\end{tabular} & 0.52 & 0.88 & no \\
losses: & 0.89 & 0.60 & no \\
\hline $\begin{array}{l}\text { Bleichrodt and Pinto [5] } \\
\text { Kilka and Weber [10] }\end{array}$ & 0.92 & 0.70 & no \\
\hline
\end{tabular}

function has to be "sufficiently concave".) However, for the classical functions used in [15] this condition has been violated in most studies, compare Table 1.

Nevertheless this result is important, in that it can be used to derive several methods to fix the problem: We have already seen one of them, namely considering bounded value functions (setting asymptotically $\alpha=0$ ). Another approach is to work with alternative weighting functions (setting for values of $F$ close to 0 and 1 the constant $\gamma=1$ ). We will explain this idea in Section 2.4.

\subsection{Proof of the general result and extensions}

In this section we give a proof of Theorem 1 and extend it to situations which are of particular interest for the classical theory of Tversky and Kahneman [15].

Proof of Theorem 1. To keep things simple we assume that $p$ is absolutely continuous, i.e. we can represent it by a finite function $p: \mathbb{R} \rightarrow \mathbb{R}_{\geq 0}$. (If this is not the case, the proof can be concluded by a simple approximation argument.)

In order to prove that $U(p)$ is finite we need to prove that it is neither $-\infty$ nor $+\infty$. For notational reasons we prove the former statement. The latter then follows by the symmetry of the problem. Thus we assume without loss of generality that $p(x)=0$ for all $x>0$.

We define a sequence $\left\{x_{i}\right\}, i=0,1,2, \ldots$ as follows:

First, let $x_{0}:=0$. Then define $x_{i}$ such that

$$
\int_{x_{i}}^{x_{i-1}} p(x) d x=2^{-i} .
$$


Since $\sum_{i=1}^{\infty} 2^{-i}=1$, we have $\lim _{i \rightarrow \infty} x_{i}=-\infty$.

The assumption that $p$ has a finite EV leads to the following estimate, using (11):

$$
\begin{aligned}
\sum_{i=1}^{\infty}-x_{i-1} 2^{-i} & =\sum_{i=1}^{\infty}-x_{i-1} \int_{x_{i}}^{x_{i-1}} p(x) d x \\
& \leq \sum_{i=1}^{\infty}-\int_{x_{i}}^{x_{i-1}} x p(x) d x \\
& =-E(p)<+\infty .
\end{aligned}
$$

Denoting $y_{i}:=-x_{i-1} 2^{-i}$ we obtain the useful estimate

$$
\sum_{i=1}^{\infty} y_{i}<+\infty
$$

We estimate the subjective utility $U(p)$ using (3) and (4). We denote by $\eta$ all terms that converge to zero as $x \rightarrow-\infty$.

$$
\begin{aligned}
U(p) & =\int_{-\infty}^{0} u(x) w^{\prime}(F(x)) p(x) d x \\
& =\int_{-\infty}^{0}-(1+\eta) u_{2} x^{\beta} w_{1} F(x)^{\delta-1} p(x) d x \\
& =\sum_{i=1}^{\infty} \int_{x_{i}}^{x_{i-1}}-(1+\eta) u_{2} x^{\beta} w_{1} F(x)^{\delta-1} p(x) d x \\
& \geq \sum_{i=1}^{\infty} \int_{x_{i}}^{x_{i-1}}-(1+\eta) u_{2} x_{i-1}^{\beta} w_{1} F\left(x_{i}\right)^{\delta-1} p(x) d x \\
& \geq \sum_{i=1}^{\infty}-(1+\eta) u_{2} x_{i-1}^{\beta} w_{1} F\left(x_{i}\right)^{\delta-1} 2^{-i} .
\end{aligned}
$$

We use the estimate

$$
F\left(x_{i}\right)=\int_{-\infty}^{x_{i}} p(x) d x=\sum_{j=j+1}^{\infty} \int_{x_{j}}^{x_{j-1}} p(x) d x=2^{-i}
$$

to obtain:

$$
U(p) \geq \sum_{i=1}^{\infty}-(1+\eta) u_{2} x_{i-1}^{\beta} w_{1} 2^{-\delta i}
$$

Using the definition of $y_{i}$ we derive

$$
U(p) \geq \sum_{i=1}^{\infty}(1+\eta) u_{2} w_{1} y_{i}^{\beta} 2^{i(\beta-\delta)} .
$$


By (12) we know that $\lim _{i \rightarrow-\infty} y_{i}=0$ and hence $y_{i}^{\beta}$ is bounded. Using the assumption $\delta>\beta$ and that $\lim _{x \rightarrow-\infty} \eta=0$, it is clear that the infinite sum in (13) converges. Thus $U(p)$ is finite.

Instead of posing conditions on the value - and the weighting functions, we can also impose conditions on the class of admissible probability distributions and in particular their decay at infinity:

Theorem 6 Let $U$ be an arbitrary CPT subjective utility with value function $u$ satisfying (3) and weighting function $w$ satisfying (4). Let $p$ be an (absolutely continuous) probability distribution such that for all $q<0$ there exists $C>0$ such that $p(x) \leq|x|^{-q}$ for all $|x| \geq C$. Then $U(p)$ is finite.

Proof. Due to the symmetry of the problem (see above), we can assume without loss of generality that $p(x)=0$ for all $x>0$. By assumption there exist $\delta \in(0,1]$ and $\beta \in(0,1)$ corresponding to (3) and (4). finiteness of $U(p)$. Define

$$
q:=\frac{\delta+\beta}{\delta}+1>0
$$

By the assumption on $p$, there exists a $C>0$ such that $p(x) \leq|x|^{-q}$ for all $|x| \geq C$. We rewrite:

$$
\begin{aligned}
U(p) & =\int_{-\infty}^{0} u(x) w^{\prime}(F(x)) p(x) d x \\
& =\underbrace{\int_{-\infty}^{-C} u(x) w^{\prime}(F(x)) p(x) d x}_{=: I_{1}}+\underbrace{\int_{-C}^{0} u(x) w^{\prime}(F(x)) p(x) d x}_{=: I_{2}} .
\end{aligned}
$$

The integral $I_{2}$ is obvious finite, hence it is sufficient to consider $I_{1}$. Since $p(x) \leq$ $|x|^{-q}$ for all $x \leq-C$, we have

$$
F(x) \leq \frac{1}{1-q}|x|^{1-q} .
$$

Using the same asymptotic estimates as in the proof of Theorem 1 we obtain

$$
\begin{aligned}
I_{1} & =\int_{-\infty}^{-C}-(1+\eta)|x|^{\beta}|x|^{(1-q)(\gamma-1)}|x|^{-q} d x \\
& =\int_{-\infty}^{-C}-(1+\eta)|x|^{\delta+\beta-1-q \delta} d x .
\end{aligned}
$$

Now, using the above definition for $q$, this simplifies to

$$
I_{1}=\int_{-\infty}^{-C}-(1+\eta)|x|^{-1-\delta} d x .
$$

Since $\delta>0$, this is integrable, and thus $U(p)$ is finite. 


\subsection{Alternative weighting functions}

Using the general results of the previous section, it is easy to suggest a new type of weighting functions that avoids infinite values for the subjective utility. By Theorem 1 we only need to find functions $w_{-}, w_{+}:[0,1] \rightarrow[0,1]$ with the following properties:

(i) $w_{ \pm}(0)=0, w_{ \pm}(1)=1$,

(ii) $w_{ \pm}$are strictly increasing on $[0,1]$. (This condition is violated for the classical weighting function (for small values of $\gamma$ ) as suggested by [15], compare Sec. 1.2.)

(iii) $w_{ \pm}$are continuously differentiable on $[0,1]$, i.e. $w_{ \pm}^{\prime}(0)$ and $w_{ \pm}^{\prime}(1)$ are finite. (This condition is violated by all usual weighting functions, e.g., [15] and [13].)

The constants $\delta, \gamma$ in Theorem 1 will then be $\delta=\gamma=1$ and the conditions $\alpha<\gamma$ and $\beta<\delta$ will be trivially satisfied since $\alpha<1, \beta<1$ by assumption. As a particular example we give a polynomial function and prove the following result:

Proposition 2 Let $a \in(0,1), b \in(0,1)$. Let the weighting function $w:[0,1] \rightarrow$ $[0,1]$ be given by

$$
w(F):=\frac{3-3 b}{a^{2}-a+1}\left(F^{3}-(a+1) F^{2}+a F\right)+F .
$$

Then $w$ satiesfies the assumptions of Theorem 1 for a finite subjective utility.

The function $w$ is not arbitrarily chosen: It is actually the simplest polynomial that satisfies all of the above conditions. It has the feature that the two parameters $a$ and $b$ have the following easy interpretation: $a$ is the point on which $w$ changes from overweighting to underweighting, i.e. where $w(a)=a$. The second parameter $b$ corresponds (like the parameter $\gamma$ in the original model) to the curvature of $w$. (One can easily see that there exists no polynomial of degree less than three which has a concave-convex structure. A standard ansatz with a polynomial of degree three then leads to the above formula for $w$.)

A one-parameter model can be obtained by assuming that $a=1 / 2$. The formula then simply reads

$$
w(F)=(4-4 b) F^{3}-(6-6 b) F^{2}+(3-2 b) F .
$$

Proof. All properties can be easily checked, since $w$ is a polynomial. (This is a big technical advantage compared to other weighting functions.) The finiteness of the subjective utility then follows immediately from Theorem 1.

In Figure 2 we present plots of $w$ for different values of $a$ and $b$. Of course the definition of the weighting function $w$ as a polynomial is just a suggestion, there are other possibilities if one allows for more complicated functions. 


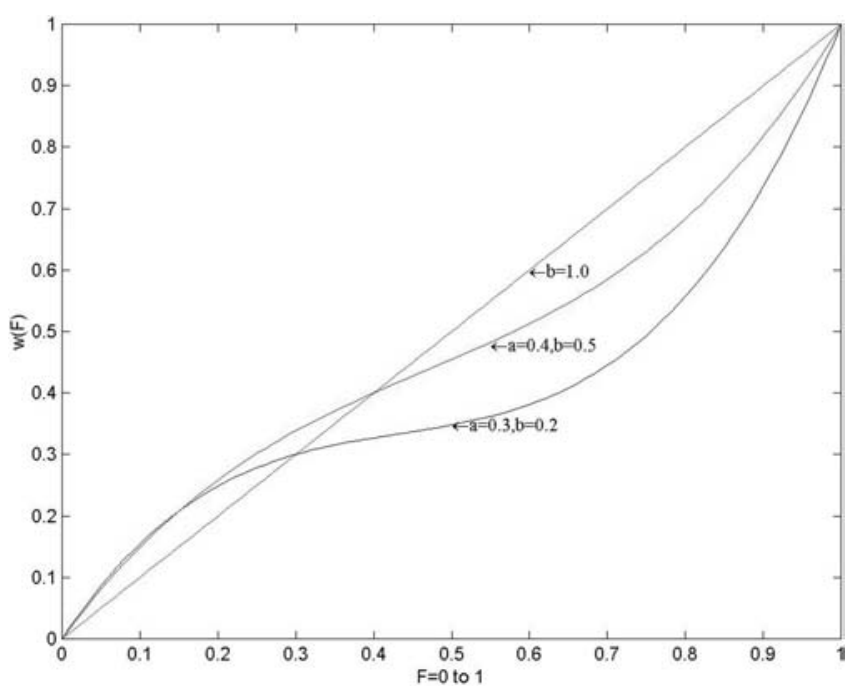

Figure 2. Alternative weighting functions $w$, avoiding the paradox of infinite subjective utility, for some choices of the parameters $a$ and $b$

\section{Conclusions}

We have seen that the standard cumulative prospect theory can lead to a strange result, namely an infinite subjective utility for a probability distribution of outcomes which has only a finite EV. To conclude, we list four possible ways to fix the problem and discuss them briefly:

1. If we allow only for probability distributions with exponential decay at infinity (or even with bounded support), the problem does not occur, as we have proved in Theorem 4 and Theorem 3. In many applications, this is the case. However, it seems to be somehow dissatisfying to work with this restriction. In particular in problems where we are interested in finding the optimal probability distribution (subject to some constraints), it might well happen that we obtain a "solution" with infinite subjective utility, compare [17].

2. It is possible to assume that $\gamma>\alpha$ and $\delta>\beta$, where $\gamma, \delta$ are the parameters of the weighting function and $\alpha, \beta$ are the growth rates of the value functions. By Theorem 1 this is sufficient to ensure finite subjective utility. Unfortunately, this assumptions seems to contradict many of the measured parameters in experiments (compare Table 1).

3. The value function can be modified for large gains and losses such that it is globally bounded. This again ensures a finite subjective utility (compare Theorem 5). There are also other theoretical reasons summarized in the survey article by Hens [8], compare also the work by De Giorgi et al. [7].

4. The final idea is to modify the weighting function $w$ as has been suggested in Section 2.4. This guarantees a finite subjective utility, independently of the choice of the value function (as long as it has a convex-concave structure). It 
is interesting to compare this approach to the weighting functions which have been obtained experimentally by Abdellaoui et al. [1].

As a last remark we mention the problem regarding the non-monotonicity of the classical weighting function by [15] which we had pointed out in Section 1.2. This problem suggests strongly to use an alternative weighting function - not necessarily the one that we introduce in Section 2.4, but, e.g., one of the already existing variants listed in Section 1.2 - in further experimental and theoretical studies.

\section{References}

1. Abdeallaoui, M.: Parameter-free elicitation of utilities and probability weighting functions. Management Science 46, 1497-1512 (2000)

2. Arrow, K.J.: The use of unbounded utility functions in expected-utility maximization: Response. The Quarterly Journal Of Economics 88, 136-138 (1974)

3. Bernoulli, D.: Specimen theoriae de mensura sortis. Commentarii Academiae Scientiarum Imperialis Petropolitanae (Proceedings of the Royal Academy of Science, St. Petersburg) (1738)

4. Blavatskyy, P.R.: Back to the St. Petersburg paradox? Management Science (to be published)

5. Bleichrodt, H., Pinto, J.L.: A parameter-free elicitation of the probability weighting function in medical decision analysis. Management Science 46, 1485-1496 (2000)

6. Camerer, C., Teck-Hua, H.: Violations of the betweenness axiom and nonlinearity in probability. Journal of Risk and Uncertainty 8, 167-196 (1994)

7. De Giorgi, E., Levy, H., Hens, T.: Existence of CAPM equilibria with prospect theory preferences. Preprint 157, Institute for Empirical Economics, University of Zurich (2004)

8. Hens, T.: Making prospect theory fit for finance. In preparation

9. Kahneman, D., Tversky, A.: Prospect theory: an analysis of decision under risk. Econometrica 47, 263-291 (1979)

10. Kilka, M., Weber, M.: What determines the shape of the proability weighting function under uncertainty? Management Science 47, 1712-1726 (2001)

11. Lattimore, P.K., Baker, J.R., Witte, A.D.: The influence of probability on risky choice. Journal of Economic Behavior and Organization 17, 377-400 (1992)

12. Menger, K.: Das Unsicherheitsmoment in der Wertlehre. Zeitschrift Nationalökonomie 51, 459485 (1934)

13. Prelec, D.: The proability weighting function. Econometrica 66, 497-527 (1998)

14. Tversky, A., Fox, C.R.: Weighing risk and uncertainty. Psychological Review 102, 269-283 (1995)

15. Tversky, A., Kahneman, D.: Advances in prospect theory: cumulative representation of uncertainty. Journal of Risk and Uncertainty 5, 297-323 (1992)

16. Wang, M., Fischbeck, P.S.: Incorporating framing into prospect theory modeling: a mixture-model approach. Journal of Risk and Uncertainty 29, 181-197 (2004)

17. Wang, M., Rieger, M.O.: Optimal financial product design under cumulative prospect theory. Work in progress

18. Wu, G., Gonzalez, R.: Curvature of the probability weighting function. Management Science 42, 1676-1690 (1996) 